\title{
1. Smart cities beyond algorithmic logic: digital platforms, user engagement and data science
}

\author{
Nicos Komninos, Anastasia Panori and \\ Christina Kakderi
}

The twenty-first century is marked by new challenges and capabilities, both defined by the progress in Internet technologies, data science, and artificial intelligence (AI). Growth patterns are changing under the influence of digitalisation, the expansion of the Service Dominant Logic (Anttiroiko and Komninos, 2019), and the rise of 'prosumer' and sharing economies (Oskam and Boswijk, 2016; Ritzer and Jurgenson, 2010). Full and stable employment probably is no longer feasible due to automation expanding by rapid advances in AI and machine learning (Brynjolfsson and McAfee, 2011). Environmental sustainability and climate change are emerging as major threats to all cities, especially those closely located to sea level (UN-DESA, 2013), while a new science of data applications provides means for better forecasting and risk management (DeLyser and Sui, 2014; Hey et al., 2009).

Cities are strongly affected by these changes. The character of urban development and planning are radically changing with the use of smart technologies and user engagement (Komninos et al., 2019). Cities are highly routinised places and many daily actions and decisions are based on standard operations in transportation, energy usage, utility networks, and location-based services. Consequently, cities offer multiple domains for pervasive application of AI, algorithms, and automation. The concept of the smart city envisages an automated future and the efficiency gains made possible by the automation of services and utilities (Batty, 2018).

The promise of smart cities is that they can address more effectively complex contemporary problems of growth and sustainability and provide more intelligent systems of decision-making and innovation. Smart cities are introducing a new paradigm of urban planning and development that combines utopian visions and practical solutions for a more sustainable, 
democratic, and cohesive future. Smart cities offer numerous solutions to improve the economy, infrastructures and utilities, the environment, and living conditions.

However, the algorithmic logic for automation and optimisation that guides smart city solutions is not enough for delivering the full potential of current advances in science and technology. This is the central argument of the book. The current hype about abundant business opportunities of smart cities requires a critical approach. Huge amounts of information are generated in cities and subjected to extensive 'big data' exploitation. Significant risks result from smart systems, especially the serious infringements of privacy rights by the gathering and exploitation of data and the autonomous operation of machines, in some cases beyond possibilities of human control. Norbert Streitz (2017) argues for the need to redefine the 'smart everything' paradigm and reconcile humans and technology, ensuring that humans remain at the control position.

Smart cities emerge from collaboration technologies (digital platforms, Internet of Things (IoT), social media, blockchain), data science, and AI. The algorithmic logic, under which these technologies operate, can be much more effective if combined with other sources of intelligence available in cities, such as human intelligence, creativity and innovation, and collective and collaborative intelligence within institutions or over platforms. Along this line of thought, the book presents a collection of chapters on this subject, organised in three parts.

Part I brings together chapters and authors that discuss the academic establishment of the smart city paradigm and the drivers of city intelligence. We are dealing with a very rapid establishment of a new paradigm with exponential annual rate of growth in publications. The chapters in Part I look at the sources and drivers of innovation and intelligence in smart cities as the outcome of collaborative endeavours rather than algorithms and automation. Part II focuses on major technologies that allow collaborative initiatives to scale-up. The chapters discuss smart cities as a technological construct driven by information technologies and embedded smart objects, but also as complex cyber-physical systems of innovation in which physical and social spaces, knowledge processes, and digital technologies are blended and produce new solutions to current challenges. Then, Part III looks into the governance of smart cities, and mainly how technologies and digital platforms allow for citizen engagement and collaboration networks to generate innovations for better cities. 


\subsection{SMART CITIES, ALGORITHMIC LOGIC AND THE QUEST FOR INTELLIGENCE}

The origin of city intelligence is the main problem discussed in Part I. What makes cities smart? What are the drivers of city smartness or intelligence? Our critical view of the smart city algorithmic logic and the risks associated with it comes from understanding city intelligence as a process of innovation shaped by the integration of human, machine, and collective intelligence.

The algorithmic logic is dominant in multiple applications for smart cities that improve traffic congestion, find parking spaces, encourage car sharing, detect and respond to accidents, optimise allocation of facilities, promote buildings and utilities efficiency, energy saving and the use of green energy, and watch and forecast environmental conditions (Gavalas et al., 2016; Zambonelli et al., 2018). Artificial intelligence is also used in city governance to restructure the relationship between citizens and bureaucracy (Anthes, 2015). Such solutions are specific per city district, sector, or problem and tend to cover the diversity of city life by accumulation of discrete solutions. In most cases, there is little interoperability between smart city solutions, which occurs at the infrastructure of sensors deployed and the cloud computing for data storage and processing. While the same algorithms are used for optimisation in different city domains and e-services, there is little communication and data exchange between solutions.

The generalisation of this algorithmic logic and the cumulative expansion of AI-based solutions to all domains of a city are associated with three main threats: (a) the transparency of the algorithmic solutions, (b) the compatibility of technological and social optimisation, and (c) the control capacity.

Currently, the most advanced algorithmic logic can be found in deep learning solutions that allow machines to learn from data. It is a fascinating idea, a machine that can learn by supervised learning methods, reinforced learning, and the backpropagation algorithm. This is a different approach from traditional AI, which focused on the processing of symbols according to rules, reasoning, and transformation of symbols through programming. The new approach is trying to solve a representation (rather than reasoning) problem (how knowledge is represented) and deploys neural networks in which the connections between neurons are not programmed but trained by data - huge amounts of data. However, how this happens is not clear; there is no transparency and causality in the connection of inputs and outputs. Stuart Russell in a recent interview to Martin Ford (2018, p. 42) points out: "We are just beginning now to get some theoretical understanding of when and why the deep learning hypothesis is correct, but to a large 
extent, it's still a kind of magic, because it didn't have to happen that way." And he continues by saying that there are some properties in the real world related to vision, sound, and speech, which can be captured by a deep learning network to provide a good prediction.

This type of 'narrow' AI, based on learning and training from data, has proved very effective and works in problems related to computer vision, speech recognition, natural language understanding, robotics, medical image analysis, and self-driving vehicles, which feed smart city solutions. But, when we move to more complex problems, for instance challenges of growth and sustainability, which are distributed and ill-defined problems, with models changing quickly over cycles and solutions depending on institutions that are not transferable from one place to another, both traditional and current AI are ineffective. In problems heavily depending on context, such as those of growth, sustainability, and poverty, the current AI is not enough, and machine intelligence should be combined with other sources of intelligence - human and organisational intelligence - to produce effective solutions. The lack of transparency is also a major barrier in public policy and big investments in cities. A correlation between input and output is not enough. More important is the justification of choices, based on theory and policy accountability.

Technological optimisation is not always social optimisation too. In smart cities we should avoid an optimisation that is merely technological, in which an algorithm defines a best solution based on data provided by the urban system. We should combine technological and social optimisation, avoiding for instance social polarisation between super-rich and super-poor, and robots replacing unskilled labour on a mass scale. We should also reject technological solutions that are not financially and environmentally sustainable. Thus, algorithmic solutions should stand on a wider intelligence base, either collective intelligence or human intelligence provided by human agents and human communities. We do not propose an Artificial General Intelligence (AGI) that could counter-balance failures of human intelligence, and in any case it is not known when and if that will become available.

Thus, in smart city solutions we should seek for integration and complementarity in heterogeneous networks between humans, communities, and machines. AI and deep learning networks can be a source of insight on how network nodes can be connected. Convolutional networks, for instance, are set by multiple layers, in which the first layer is organised by a convolution principle, the second by a non-linearity principle, and the third by a pooling principle (see LeCun, in Ford, 2018). Thus, in complex challenges of cities, addressed with smart city solutions, a key concern is about the way heterogeneous networks are set, enabling the advancement 
of further human and community intelligence through digital platforms, data and artificial intelligence.

The control capacity of algorithmic solutions is also a topic widely discussed in the literature as a serious threat. It concerns both the current state of AI, self-driving cars and fatal accidents or the Boeing 737 Max disasters (Arumugham, 2019; Fischer, 2019), and future AGI (Bostrom, 2014, 2017; Häggström, 2019; Müller, 2016; Torres, 2019). Pure algorithmic solutions, based on machine decision-making without validation and human control, bring new kinds of extreme risks. Cities as a system of systems are characterised by enormous complexity, and the interdependence of urban sub-systems makes it impossible to switch off something if another sub-system goes wrong. In fully automated systems, the partial objectives of a sub-system will not be aware of higher order objectives and needs.

Our view of smart cities goes beyond algorithmic solutions and focal system optimisation, without underestimating the importance and contribution of such solutions. Our intention is to make clear the difference between smart city optimisation and smart city intelligence. The main argument of the book is that solutions for smart cities, addressing the current challenges of cities, cannot be based on an algorithmic logic only. Instead, they should use more advanced heuristics generated by the engagement of citizens, communities, and institutions. As we have explained in other publications (Komninos, 2014), smart cities set in motion three novel innovation circuits: providing e-services (c1), improved decision-making and investment systems (c2), and optimised user behaviour (c3). Digital platforms and online collaboration networks into urban ecosystems allow for these innovation circuits to appear and operate. This understanding of smart cities as systems generating intelligence, in the full meaning of the term, is presented in Part I with four chapters.

Chapter $\mathbf{2}$ is a critical reflection upon the first three decades of research on smart city development and presents a series of divisions affecting this fast-emerging knowledge domain. The chapter is based on a group of studies that the authors have undertaken between 2017 and 2018 in order to investigate the mechanisms of knowledge production shaping the intellectual structure of smart city research. These studies and their findings are examined, and a synopsis is offered, which captures the significance of the insights into the current state of smart city research. Five development paths are identified: experimental solutions and smart cities as testbeds for IoT solutions, the ubiquitous city path, the corporate smart city model, the European smart city for a low-carbon economy, and a holistic path connecting digital, intelligent, smart dimensions of cities. A main conclusion is the identification of multiple divisions and the absence of consensus in regard to effective management for smart cities, a condition which undermines smart city practice and the potential to achieve urban sustainability. 
Chapter 3 looks into the algorithmic city and the transformation in politics, governance, and service provision. Technological development has had a dramatic impact on the nature of urban life, and the pace of change is likely to continue to be fast for the foreseeable future. This chapter draws attention to fundamental changes in the logic of urban life affected by technologically enhanced disembedding mechanisms, examples of which include a range of autonomous service, learning, interactive, and control systems. The chapter conceptualises the fundamental dimensions and emerging forms of algorithm-based practices in local public affairs. The discussion starts with a contextual description of the algorithmic revolution. It then concretises this idea by focusing on the use of algorithms in politics, regulation, governance, and service provision, and lastly it exemplifies real-life developments by presenting a few cases from the USA. Paradigmatic examples include the use of algorithms in law enforcement and eligibility systems, which have a profound impact on people's lives. Such systems have the undeniable potential to smarten up decision-making and governance processes, but they may at the same time increase inflexibility, opacity, and bias. Therefore, an emerging algorithmic city must be guided by carefully crafted policies and governance principles that provide a sufficient degree of flexibility, transparency, and democratic control.

Chapter 4 discusses smart cities as ecosystems of innovation and experimental environments for user-driven innovation. The concept of smart cities is a response to the challenges faced by cities to meet broadly shared societal objectives regarding socio-economic development, quality of life, and resilience. The promise of the smart city concept is to create an environment of open, participative and citizen-centric innovation based on sharing of knowledge and resources for experimenting with innovative solutions for urban development. The last decade witnessed a wide range of smart city concepts, experiments, and practices, balancing between technological and social innovation perspectives and between corporate and citizen-centric stakeholder orientations. There is a growing emphasis on creating and nurturing urban innovation ecosystems comprising an environment of cooperation, experimentation, and learning rather than planning and implementation of smart city solutions. Realising the promise of the smart city vision requires creating open, participative, and sustainable forms of cooperation among stakeholders to breed and nurture such ecosystems as key infrastructures of urban development.

Chapter 5 focuses on the creation of intelligence in smart cities. Smart cities offer a suitable environment for the creation of smart ecosystems that are characterised by a digital, therefore, non-spatial connectivity. In this context, platforms act as facilitators producing externalities similar to spatial agglomerations, resulting in the creation of smart ecosystems, 
reinforcing aspects of knowledge exchange and collaboration. In this chapter, we have identified a four-layer structure of intelligence that is generated in smart cities, including: (i) human intelligence encompassing aspects of human problem-solving abilities and social interaction; (ii) artificial intelligence working complementary to human capabilities, encompassing data collection and mining processes and resulting in higher information processing compared to traditional methods; (iii) collective intelligence which is generated through the population of digital platforms; and (iv) collaborative intelligence referring to open innovation, co-creation and co-design. In this regard, decision-making and city governance should be the outcome of well-coordinated interactions between datasets, analytics, platforms, and actors, each one of them contributing in a different way to the overall problem-solving process. It becomes evident that smart cities create a connected intelligence space, including dimensions of 'human ability', 'artificial modelling', 'collectiveness', and 'collaboration' that produce awareness, synergies, and positive externalities. Within this framework, companies and organisations, instead of being part of an established physical ecosystem, have the capacity to build their own smart ecosystem.

\subsection{SMART CITIES AT THE CROSSROADS OF IOT, SOCIAL MEDIA AND DATA SCIENCE}

Following Wolfram's (2002, p. 130) idea that "It is possible to make things of great complexity out of things that are very simple", Part II of the book aims at providing insights regarding the ways in which different infrastructures and individual actors within a smart city can be connected towards optimised knowledge production and activities. The chapters share the idea that collaborative intelligence is about the whole being more than the sum of its parts (Eberhart et al., 2001). Thus, empowerment of actors' connectivity, through emerging digital technologies, is crucial towards providing a fertile ground for knowledge discovery, learning, and production of intelligence.

All the chapters included in Part II point out that there is a strong connection between smart cities and the evolution of digital technologies, including IoT, social media, and data science. Innovative features of digital technologies affect in various ways actors' connectivity within a smart city, and thus create novel channels for interactions, not only between individuals, but also between local authorities, researchers, and businesses. The diffusion of disruptive IoT technologies changes traditional channels of information collection and sharing, as well as the decision-making processes, promoting broad collaboration among local stakeholders. 
Multi-user interactions can be strengthened on multiple levels. Interoperability between different devices, networks, and data within smart cities is a key element that optimises data collection and processing, coming from various sources, such as users, sensors, and the web. More specifically, Part II investigates four emerging areas powered by the growing use of cloud services, social media applications, blockchain technology and data analytics, combined with recent advances in low-cost hardware and peer-to-peer technologies. It investigates novel approaches focusing on harnessing the power of data generated in smart cities, by strengthening actors' connectivity and collaboration. Advances in digital technologies have played an essential role in this direction, boosting interoperability of devices, information sharing, and security and robustness of information.

To this end, investigation of novel cloud services focusing on enabling traditional city entities (node, user, and provider) to be exposed and consumed as a service is an essential aspect (Loscri et al., Chapter 6 this volume). Multi-disciplinary data collection and processing in smart cities offer a continuously growing ability to capture information about cities, giving designers and technologists a unique opportunity to understand, improve, and invent spatial dynamics (Zhang et al., Chapter 9 this volume). At the same time, the evolution of new frameworks of interaction and multi-stakeholder collaboration between administrative actors and citizens is of key importance, due to the penetration of social media into day-today operations (Vakali and Moustaka, Chapter 7 this volume). However, security aspects related to this continuously growing citizen-government interaction should be further strengthened, ensuring the legitimacy and accountability of city operations. In this regard, blockchain technology could take established services, processes, regulations, and policies to the next level by providing a secure decentralised environment (Tsampoulatidis et al., Chapter 8 this volume).

Chapter 6 focuses on investigating the essential technological requirements for a smart city. The authors identify the main technical challenges in the heterogeneity of devices, data, networks, protocols, and standards that need to be addressed towards implementing the smart city vision. To overcome these limitations, they propose the Cloud of Meshed Cooperative heterogeneous Things (CoMCoT), which enables every traditional city's entity (node, user, and provider) to be exposed and consumed as a service. Beyond traditional IoT services, such as data abstraction and mutualisation, the CoMCoT provides more holistic functionalities by targeting full interoperability. The chapter identifies the main technical challenges and some interesting use cases. First, it describes the Cloud of Things and its potential limitations when applied to a smart city environment. Given these limitations, the authors introduce their vision of the CoMCoT, and 
thoroughly describe its main requirements, including functionalities, tools, and standards, towards realising that vision. Data collection and processing, knowledge representation and standardisation are key elements of this vision. In addition to technical challenges, the authors point out a set of social challenges that also need to be addressed throughout the smart city context, including participation of multiple stakeholders with different or even conflicting interests, as well as the involvement of municipalities. Finally, Chapter 6 concludes by highlighting the shift towards a new concept, wider than the traditional Cloud of Things, introducing the CoMCoT, where sensors and actuators not only can be discovered and aggregated, but also dynamically act as service providers.

Chapter 7 provides insights regarding the ways in which the penetration of social media and various digital technologies into daily life empowers both administrative actors and citizens to create new frameworks for interaction and multi-stakeholder collaboration. The authors argue that this effectively contributes to the generation, acquisition, and diffusion of information, which is crucial for exploring urban dynamics and providing an efficient monitoring and management mechanism for cities. This chapter deals with the user-generated content (UGC) through new technologies and investigates aspects for its exploitation towards achieving the smart city vision. The authors indicate that active citizenship and UGC exploitation play a decisive role in shaping smart cities, as well as in tracking and forecasting the evolution of urban potential. The chapter analyses the three user-centric ways in which citizens participate in the UGC creation and presents a systematic literature review which concerns the exploitation of UGC in smart cities over the past twenty years. The findings reveal that UGC was utilised to address a variety of urban issues such as detection of dense areas and land uses, urban planning, citizen engagement, tourism, ride and car sharing, advertising, marketing, and more. Moreover, results indicate that the most popular means of creating UGC are mobile phones and opportunistic sensor networks, and that opportunistic and participatory sensing are the most popular forms of people participation in UGC creation. Several methods for data analysis are exploited depending on the type of data and the purpose of the studies in which they were used. Moreover, a novel framework for tourism and road passenger transport is proposed, which aspires to exploit UGC shared by users during both their direct and asynchronous communication aiming at promoting tourist destinations and designing quality, economic, and personalised transport services for travellers.

Chapter 8 explores the role of Information and Communication Technology (ICT) for enabling the digital transformation of established public services, processes, and policies in smart cities. The authors point 
out that this transformation process started from e-Gov 1.0 initiatives and spans to the e-Gov 3.0 phase with special focus on interconnected citizens, smart and interconnected devices, big data analytics, and cloud computing. As the smart city initiative flourishes there is an overwhelming need for robust, secure, and flexible solutions that will pave the way to citizens' participation and inclusion. The chapter proposes the adoption of a blockchain framework, which will not only facilitate the acceptance of new governance models from the official stakeholders, but will also dynamically identify, create, and propose new policies, regulations, and initiatives according to social trends and the citizens' maturity level. This framework takes into consideration social, environmental, and economic aspects, as well as established policies and good practices for proposing solutions to stakeholders. The user-centric ways in which users participate in the creation of content are discussed, while a systematic literature review summarises the issues that have been addressed through the exploitation of user-generated content, the means used for its creation, and the methods used for its analysis.

Chapter 9 points out that designers and technologists have the unprecedented opportunity to understand, improve, and invent spatial dynamics, given the growing ability to capture information about cities and their constituents. The authors focus on the Underworlds Project developed by different laboratories at the Massachusetts Institute of Technology, in collaboration with the Kuwait government. It is an interdisciplinary approach, combining robotics, bioengineering, and genomics to paint a microbial portrait of the urban environment, based on large data collection processes. More specifically, the bespoke network of automatic samplers works in the sewage to collect data about health trends at a high spatial and temporal resolution, which gives researchers tools to analyse the many facets of urban dwelling and public health. At the same time, with mounting public concerns over data privacy, smart city endeavours, such as Underworlds, are facing a critical juncture in their evolution. With a sensible eye towards how these endeavours respond to and evolve with the blurring boundaries between public and private, they allow cities and citizens to collectively harness the boundless opportunities that a smart city offers.

\subsection{SMART CITIES, PARTICIPATORY GOVERNANCE AND DIGITAL PLATFORMS}

At the end of the day, the making of smart cities is closely related to planning and governance: how we use digital technologies, connect resources 
of intelligence, and address the complex challenges of contemporary cities. Thus, Part III of the book focuses on these issues. The questions raised concern (a) how smart city planning and governance contribute to connectivity and integration of human, collective, and artificial intelligence that change the routines of urban activities and the socio-economics of cities, and (b) how planning and governance deploy networking technologies, social media connecting people, IoT connecting devices and smart objects, blockchain connecting individuals and institutions, data and analytics connecting activities and different fields of science and technology, to produce innovative solutions. In dealing with these questions, core concepts are those of participatory governance and planning with digital platforms.

Smart city planning is not planning as usual - top-down, rigid, and state-driven. It is a collective and collaborative endeavour rather than the solid outcome of a planning institution. Many initiatives and applications start from citizens, small companies, and non-profit organisations. Their engagement creates diversity and complexity. Cities are complex systems and digital technologies increase their complexity. Rapidly evolving digital technologies and participatory governance make the smart city planning fuzzy and market-driven. Master plans, the dominant city planning method for more than a century, are quite inadequate to capture the rhythm and volatile nature of smart city solutions. The concept of 'planning without plans' refers to a smart city that is gradually shaped by evolving technologies and windows of opportunities that are opened by city stakeholders (Komninos et al., 2019).

Smart city planning and governance do not deal with technology only. The city is primarily a socio-economic entity and this character remains dominant in smart cities too. In parallel to planning for connectivity and e-services, smart cities should address social divides and disparities, poverty, discrimination, and socio-economic inequality. In fact, success over these challenges can prove that smart cities are really entities of higher intelligence, capable of resolving problems that traditional cities are unable to address.

In this regard, there is an organisational concept that has proven particularly successful in collaborative smart city development: the digital platform. A platform is a space over which people stand to make something better. Platforms illustrate the concept that 'dwarfs standing on the shoulders of giants can see further'. "If I have seen further than others, it is by standing upon the shoulders of giants" is a quote attributed to Isaac Newton. There is long tradition of manufacturing platforms offering internal economies of scope (Gawer and Cusumano, 2014). A digital platform is different. It is hardware or software addressed to external parties, used to make or host applications, datasets, and e-services. Koskinen et al. (2018) 
identified two types of digital platforms with transactional and innovation functions. Digital transactional platforms facilitate exchanges and trade. Digital innovation platforms facilitate the production of digital assets, content, software, e-services, and commons. Good examples are iOS and Android that facilitate third-party developers to build applications for smart phones. Most successful IT companies of the current growth cycle are platform-based companies. The entire sharing economy is based on platforms and the engagement of producers and users over platforms.

The success of digital platforms is attributed to multi-sided markets based on value-creating interactions between external producers and consumers. In such markets, the platform becomes the core mediator between the multiple sides of the market. In simple words, in multi-sided markets, buyers and sellers cannot find each other and platforms offer an externality facilitating this matching.

In smart city planning, digital platforms offer modularity and standardisation over which large-scale network effects take off. Platforms operate as public goods, even when their managing body is a private organisation. They operate as goods with non-rivalry and non-exclusivity. But beside their significance for the deployment of smart cities, digital platforms are unable to smooth market transactions and remove all tensions with incumbent companies (Nicholson et al., 2019). The quest for regulation addressing market failure remains alive.

Digital platforms make possible new forms of smart city planning: connecting citizens, turning consumers into producers, introducing radical cultural change along the 'zero' culture minimising or eliminating various threats, and making the smart city an outcome of collaboration and collective intelligence. Thus, looking at smart cities from this participatory and platform-based perspective, Part III of the book includes four chapters on planning, policy, and governance.

Chapter 10 investigates further the smart city aspects that tackle physical structures, such as spatial planning, housing, and regeneration of historical areas, which have been largely understudied. Out of the currently existing smart cities, most are insufficiently adapted to their context. Addressing these shortcomings, the authors identify the possible classifications and typologies of spatial planning for smart city development by means of case study research. The research focuses on 'star' cases of smart cities, particularly Vienna, Thessaloniki, Stockholm, India's smart cities, Multimedia Super Corridor, Heraklion, and Amsterdam. The study identifies two typologies of spatial planning in a smart city framework. The first one follows a spatial planning approach based on urban characteristics, and includes classifications based on urban features, neighbourhood character, and technical infrastructure. The second typology uses a spatial planning 
approach based on scale and outlines classifications at national scale, regional/metropolitan scale, municipality/local scale, and distributed and project-based scale.

Chapter 11 points out that while social policy aims to tackle socioeconomic inequalities and the distribution of quality of life on a wider social base, and thus may be seen as an important tool for preventing or interfering with the negative effects of the digital divide (and other social disparity situations), it has not been sufficiently dealt with in the existing smart city literature. The chapter provides a better understanding of the technology-based issues of smart cities and the position of social groups most affected by these developments. The scope of this research covers individuals who live in smart cities and face a 'digital divide' due to their individual or contextual characteristics. In this context, variables such as age, income, education level, gender, or mental and physical health put some individuals in a relatively disadvantaged position in terms of their ability to access and use digital technology. The authors develop a conceptual framework for mapping out the forces at work and argue that digital technologies can be more accessible and usable for more city residents through social policy. Moreover, the implementation of such a strategy would lead to an increase in the quality of urban life and can be shared by more people in smart cities.

Chapter 12 analyses how the concept of 'sharing' relates to the smart city paradigm. Theories on 'sharing' and on smart cities have discursive resemblances, as both seek to connect communities, to empower residents, and to promote the efficient use of resources. However, if we look at the actual practice of what is known as 'sharing', rather than taking the prescriptive perspective of what it ought to be, this practice may contradict its original inspiration and, therefore, the purpose of smart cities. The chapter looks at the origins and development of Uber and Airbnb, and analyses their growth dynamics as 'multisided platforms' as well as their influence on incumbents and their negative externalities. The conclusion is that digital platforms rely on secrecy as a strategic weapon, and that they disempower citizens by contributing to the commodification of their neighbourhoods.

Chapter 13 focuses on different 'zero initiatives' that have recently emerged in smart cities. By investigating the main components of three different zero-related policies - zero fatal traffic accidents, zero crime, and zero waste - it identifies areas of common ground and establishes a conceptual and practical integration of 'vision zero'. The chapter shows that although the core elements of such strategies are rooted in the intersection and developments of specific science and policy areas, they all share some common principles and methodological concepts. The systemic, complex, and ambitious character of vision zero strategies, coupled with smart 
city technologies and infrastructure, provide significant added value to traditional solutions and efforts in dealing with urban problems. By combining these two discourses, the chapter finds connections between smart city developments and vision zero initiatives to facilitate their diffusion to different urban settings and city domains.

All in all, the chapters in this book articulate a process of smart city creation and effectiveness that is based on collaborative technologies, participatory governance, and emergence of intelligence through collaboration and integration of distributed capabilities in heterogeneous systems.

\section{REFERENCES}

Anthes, G. (2015). Estonia: a model for e-government. Communications of the ACM, 58(6), 18-20.

Anttiroiko, A.V. and Komninos, N. (2019). Smart public services: using smart city and service ontologies in integrative service design. In: M.P. Rodriguez Bolivar (ed.), Setting Foundations for the Creation of Public Value in Smart Cities (pp. 17-48). Public Administration and Information Technology, vol. 35. Cham: Springer.

Arumugham, V. (2019). Vaccine safety: learning from the Boeing 737 MAX disasters. Forthcoming.

Batty, M. (2018). Artificial intelligence and smart cities. Environment and Planning B: Urban Analytics and City Science, 45(1), 3-6.

Bostrom, N. (2014). Superintelligence: Paths, Dangers, Strategies. Oxford: Oxford University Press.

Bostrom, N. (2017). Superintelligence: Paths, Dangers, Strategies, revised edition. Oxford: Oxford University Press.

Brynjolfsson, E. and McAfee, A. (2011). Race Against the Machine. Lexington, KY: Digital Frontier Press.

DeLyser, D. and Sui, D. (2014). Crossing the qualitative-quantitative chasm III: enduring methods, open geography, participatory research, and the fourth paradigm. Progress in Human Geography, 38(2), 294-307.

Eberhart, R.C., Shi, Y., and Kennedy, J. (2001). Swarm Intelligence. San Francisco, CA: Morgan Kaufmann.

Fischer, D. (2019). Was Boeing's Compensation Committee sufficiently independent in judging the business risk of the 737 Max? Available at SSRN: http://dx.doi.org/ $10.2139 /$ ssrn.3370066.

Ford, M. (2018). Architects of Intelligence: The Truth about AI from the People Building It. Birmingham: Packt Publishing.

Gavalas, D., Konstantopoulos, C., and Pantziou, G. (2016). Design and management of vehicle-sharing systems: a survey of algorithmic approaches. In: M.S. Obaidat and P. Nicopolitidis (eds), Smart Cities and Homes: Key Enabling Technologies (pp. 261-89). Cambridge, MA: Morgan Kaufmann.

Gawer, A. and Cusumano, M.A. (2014). Industry platforms and ecosystem innovation. Journal of Product Innovation Management, 31(3), 417-33.

Häggström, O. (2019). Challenges to the Omohundro-Bostrom framework for AI motivations. Foresight, 21(1), 153-66. 
Hey, T., Tansley, S., and Tolle, K. (eds) (2009). The Fourth Paradigm: Data-Intensive Scientific Discovery. Redmond, WA: Microsoft Research.

Komninos, N. (2014). The Age of Intelligent Cities: Smart Environments and Innovation-for-All Strategies. London: Routledge.

Komninos, N., Kakderi, C., Panori, A., and Tsarchopoulos, P. (2019). Smart city planning from an evolutionary perspective. Journal of Urban Technology, 26(2), $3-20$.

Koskinen, K., Bonina, C., and Eaton, B. (2018). Digital platforms in the global south: foundations and research agenda. University of Manchester Centre for Development Informatics working paper. Available at https://diodeweb.files.word press.com/2018/09/digital-platforms-diode-paper.pdf.

Müller, V.C. (ed.) (2016). Risks of Artificial Intelligence. Boca Raton, FL: CRC Press.

Nicholson, B., Nielsen, P., Saebo, J., and Sahay, S. (2019). Exploring tensions of global public good platforms for development: the case of DHIS2. In: P. Nielsen and H.C. Kimaro (eds), Information and Communication Technologies for Development: Strengthening Southern-Driven Cooperation as a Catalyst for ICT4D. 15th IFIP WG 9.4 International Conference on Social Implications of Computers in Developing Countries, ICT4D 2019, Dar Es Salaam, Tanzania, May 1-3, 2019, Proceedings, Part I (pp. 207-17). Cham: Springer.

Oskam, J. and Boswijk, A. (2016). Airbnb: the future of networked hospitality businesses. Journal of Tourism Futures, 2(1), 22-42.

Ritzer, G. and Jurgenson, N. (2010). Production, consumption, prosumption: the nature of capitalism in the age of the digital 'prosumer'. Journal of Consumer Culture, 10(13), 13-36.

Streitz, N. (2017). Reconciling humans and technology: the role of ambient intelligence. In: A. Braun, R. Wichert, and A. Mana (eds), Ambient Intelligence: 13th European Conference, AmI 2017 Proceedings (pp. 1-16). Cham: Springer.

Torres, P. (2019). The possibility and risks of artificial general intelligence. Bulletin of the Atomic Scientists, 75(3), 1-4.

UN-DESA (2013). World Economic and Social Survey 2013: Sustainable Development Challenges Post 2015. New York: United Nations.

Wolfram, S. (2002). A New Kind of Science. Champaign, IL: Wolfram Media.

Zambonelli, F., Salim, F., Loke, S.W., De Meuter, W., and Kanhere, S. (2018). Algorithmic governance in smart cities: the conundrum and the potential of pervasive computing solutions. IEEE Technology and Society Magazine, 37(2), $80-87$. 